\title{
SYMMETRY FOR EXTERIOR ELLIPTIC PROBLEMS AND TWO CONJECTURES IN POTENTIAL THEORY
}

\author{
Boyan SIRAKOV \\ Laboratoire d'Analyse Numérique, Université Paris 6, Tour 55-65, 5e étage, \\ 75252 Paris Cedex 05, France \\ Manuscript received 1 October 1999
}

\begin{abstract}
In this paper we extend a classical result of Serrin to a class of elliptic problems $\Delta u+f(u,|\nabla u|)=0$ in exterior domains $\mathbb{R}^{N} \backslash G$ (or $\Omega \backslash G$ with $\Omega$ and $G$ bounded). In case $G$ is an union of a finite number of disjoint $C^{2}$-domains $G_{i}$ and $u=a_{i}>0, \partial u / \partial n=\alpha_{i} \leqslant 0$ on $\partial G_{i}$, $u \rightarrow 0$ at infinity, we show that if a non-negative solution of such a problem exists, then $G$ has only one component and it is a ball. As a consequence we establish two results in electrostatics and capillarity theory. We further obtain symmetry results for quasilinear elliptic equations in the exterior of a ball.
\end{abstract}

(C) 2001 L'Association Publications de l'Institut Henri Poincaré. Published by Elsevier B.V. All rights reserved

RÉSUMÉ. - Nous étendons un résultat classique de Serrin à des problèmes elliptiques $\Delta u+f(u,|\nabla u|)=0$ dans des domaines extérieures du type $\mathbb{R}^{N} \backslash G$ (où $\Omega \backslash G$, avec $\Omega$ et $G$ bornés). En supposant que $G=\bigcup_{i=1}^{k} G_{i}$, où $G_{i}$ sont des domaines disjoints de classe $C^{2}$, et que $u=a_{i}>0, \partial u / \partial n=\alpha_{i} \leqslant 0$ sur $\partial G_{i}, u \rightarrow 0$ à l'infini, nous montrons que ce problème admet une solution classique seulement si $G$ a une seule composante connexe et $G$ est une boule. Comme conséquence nous obtenons deux résultats sur des problèmes provenant de l'électrostatique et de la théorie de la capillarité. Nous obtenons aussi des résultats de symétrie pour des équations elliptiques quasi-linéaires dans l'extérieur d'une boule.

(C) 2001 L'Association Publications de l'Institut Henri Poincaré. Published by Elsevier B.V. All rights reserved

\section{Introduction and main results}

This paper is a contribution to the study of symmetry properties of non-negative solutions of elliptic partial differential equations, which started with the classical papers by Serrin and by Gidas, Ni and Nirenberg ([13] and [6]; see also [4] for a more recent approach). We concentrate on exterior and annuli-like domains, possibly multiplyconnected, with different boundary conditions on each connected component of the boundary.

Consider the model problem

$$
\begin{cases}\Delta u+f(u)=0 & \text { in } \mathbb{R}^{N} \backslash G, \\ u \geqslant 0 & \text { in } \mathbb{R}^{N} \backslash G, \\ u \rightarrow 0 & \text { as }|x| \rightarrow \infty\end{cases}
$$


where $f \in C^{1}([0, \infty))$, with $f^{\prime}(0)<0$. We suppose that

$$
G=\bigcup_{i=1}^{k} G_{i},
$$

where $k \in \mathbb{N}$ and $G_{i}$ are bounded $C^{2}$-domains such that $\overline{G_{i}} \cap \overline{G_{j}}=\emptyset$ for $i \neq j$.

The boundary conditions that we impose on $\partial G$ are the following

$$
u=a_{i}>0 \quad \text { and } \quad \frac{\partial u}{\partial n}=\alpha_{i} \leqslant 0 \quad \text { on } \partial G_{i}, \quad i=1, \ldots, k,
$$

where $a_{i}, \alpha_{i}, i=1, \ldots, k$, are constants and $n$ denotes the inward normal to the boundary of $\Omega \backslash G$.

Our main result (Theorem 2 below), applied to problem (1), gives the following statement.

THEOREM 1. - Suppose $u \in C^{2}\left(\mathbb{R}^{N} \backslash G\right)$ is a solution of (1), satisfying (3). Then $G$ has only one connected component (i.e. $k=1)$. Moreover, $G$ is a ball and the solution $u$ is radial with respect to the center of this ball.

Let us now describe the general setting that we consider. We study boundary-value problems of the type

$$
\begin{cases}Q u+f(u,|\nabla u|)=0, u \geqslant 0, u \in C^{2} & \text { in } \overline{\Omega \backslash G}, \\ \text { Boundary Conditions } & (\mathrm{BC}),\end{cases}
$$

where $Q$ is a (nonlinear) strongly elliptic operator, $\Omega \subset \mathbb{R}^{N}, N \geqslant 2$, is a $C^{2}$-domain, $G$ is as in (2), $\bar{G} \subset \Omega$ and $\Omega \backslash G$ is connected.

We distinguish two cases for $\Omega$.

Case A. $\Omega$ is bounded. Here the boundary conditions on $\partial \Omega$ read

$$
u=0 \quad \text { and } \quad \frac{\partial u}{\partial n}=\beta \quad \text { on } \partial \Omega
$$

where $\beta$ is a constant.

Case B. $\Omega=\mathbb{R}^{N}$. Then we suppose that

$$
\nabla u(x) \rightarrow 0 \quad \text { and } \quad u(x) \rightarrow 0 \quad \text { as }|x| \rightarrow \infty .
$$

In the particular case when $f$ does not depend on $|\nabla u|$, instead of (6) we only suppose that

$$
u(x) \rightarrow 0 \quad \text { as }|x| \rightarrow \infty .
$$

A positive solution satisfying (7) is often called a ground state.

We consider the following assumptions on the elliptic operator and the function $f$. 
(q) $Q u=\operatorname{div}(g(|\nabla u|) \nabla u)$, where $g \in C^{2}([0, \infty)), g(s)>0$ and $(s g(s))^{\prime}>0$ for all $s \geqslant 0$. In other words, we suppose that $Q$ is a regular strongly elliptic operator.

(f) $f(u, p)$ is a locally Lipschitz continuous function in $[0, \infty)^{2}$ and, in Case $\mathrm{B}$, is a non-increasing function of $u$ for small positive values of $u$ and $p$.

These assumptions are satisfied in the applications we present below. Our results remain true for any generalisation of (q) and (f) which leads to a "good" equation for the comparison function in the "moving planes" method (see Step 1 in Section 2.2). In particular, a natural question is whether we can consider nonlinearities which are not Lipschitz continuous in $u$ at the origin. See Remark 2 at the end of this section.

In the sequel we denote by (BC) either (3) and (5), or (3) and (6), or (3) and (7), depending on the case we consider. The following theorem contains our main result.

THEOREM 2. - Suppose (q) and (f) hold. If $u$ is a solution of (4) satisfying (BC), then $k=1, \Omega$ and $G$ are concentric balls centered at some point $x^{0} \in \mathbb{R}^{N}, u$ is radial, that is $u=u\left(\left|x-x^{0}\right|\right)$, and

$$
\frac{d u}{d r}<0 \quad \text { for } r=\left|x-x^{0}\right| \in\left(\rho_{1}, \rho_{2}\right),
$$

where $\rho_{1}$ and $\rho_{2}$ denote the radii of $G$ and $\Omega\left(0<\rho_{1}<\rho_{2} \leqslant \infty\right)$.

The presence of assumptions on both the solution and its normal derivative on the boundary makes problem (4) overdetermined. It can be viewed as a free boundary type problem. This kind of problem was introduced in Serrin's classical paper [13], where he considered the case $u>0, G=\emptyset, \Omega$ bounded.

In recent years there have been some partial results for non-empty $G$ and unbounded $\Omega$. In particular, when $\Omega$ is bounded, Alessandrini [1] obtained Theorem 2 when $f \equiv 0$. In the same case ( $\Omega$ bounded), Willms, Gladwell and Siegel [15] obtained the result for $f \equiv 1$ and $Q=\Delta$, provided $N=2, \Omega$ and $G_{i}$ are convex and satisfy some additional curvature conditions. The most general previous results were derived by Reichel [10-12]. He proved Theorem 2 under the additional hypotheses

$$
a_{1}=\cdots=a_{k}=a \quad \text { and } \quad 0<u<a \quad \text { in } \Omega \backslash G .
$$

Notice that in our result $(\mathrm{r})$ is not assumed a priori, but is rather derived as a consequence of Theorem 2.

The statement of Theorem 2 thus unifies and extends all the above results for problem (4).

Another related result was derived by Aftalion and Busca [3]. Using a method based on the Kelvin transform in exterior domains, they obtained Theorem 1 ( $\Omega=\mathbb{R}^{N}, Q=$ $\Delta$ ) for a different class of functions $f$, including power nonlinearities like $u^{p}$, for $\frac{N}{N-2} \leqslant p \leqslant \frac{N+2}{N-2}$. Aftalion and Busca suppose $k=1$ and $0 \leqslant u \leqslant a$. One can see, using their method together with our approach here, that their result holds if in their work the latter two hypotheses are replaced by (2) and (3).

As a consequence of Theorem 2, we obtain two results in electrostatics and capillarity theory. These problems have been open for some time.

THEOREM 3. - Two or more $C^{2, \alpha}$-regular conducting bodies in $\mathbb{R}^{N}$ do not admit constant equilibrium charge distributions on their boundaries. 
THEOREM 4. - Two or more $C^{2, \alpha}$-regular solid cylinders, dipped into an infinite plain liquid reservoir, cannot raise a capillary surface to constant heights on their walls.

As far as Theorem 3 is concerned, we recall that if $\Omega$ is a domain in $\mathbb{R}^{N}$, a charge distribution $\rho \in C(\partial \Omega)$ is said to be in equilibrium if the induced single-layer potential

$$
\psi(x)=\int_{\partial \Omega} \rho(y) \gamma(|x-y|) d \sigma_{y}
$$

is constant in $\Omega$; here $\gamma(t)=-\frac{1}{2 \pi} \log t$ if $N=2$ and $\gamma(t)=-\frac{1}{4 \pi t}$ if $N=3$. Then the function $\psi$ satisfies an equation of type (4) with $Q=\Delta$ and $f=0$. Note that when $N=2$ we have $\psi \rightarrow-\infty$ as $|x| \rightarrow \infty$, but this creates no problems when we apply Theorem 2 (see a remark by Reichel in [11]).

The physical setting described in Theorem 4 leads to an equation of type (4), with

$$
g(s)=\frac{1}{\sqrt{1+s^{2}}} \quad \text { and } \quad f(u)=-\kappa u, \quad \kappa>0
$$

(the original discussion on this problem can be found in [13]; see also [11] and [12], where the cases of one conducting body or one cylinder were studied).

Our next result concerns the case when $\Omega$ or $G$ (or both) is a priori supposed to be a ball. In this situation, to obtain a symmetry result on the solution, we do not need to assume that its normal derivative is constant on the corresponding boundary. A previous result in this sense was obtained by Reichel, who considered the case when $\Omega$ is bounded, and assumed condition (r) (with weak instead of strict inequalities, see [10]).

THEOREM 5. - Suppose G is a ball. Then the conclusion of Theorem 2 holds true if (3) is replaced by

$$
u=a>0 \quad \text { and } \quad \frac{\partial u}{\partial n} \leqslant 0 \quad \text { on } \partial G .
$$

Analogously, if $\Omega$ is a ball, the same conclusion holds if(5) is replaced by

$$
u=0 \quad \text { on } \partial \Omega .
$$

There is no difficulty in extending Theorem 5 (with the same proof) to the limiting case $G=\left\{x_{0}\right\}$ (i.e. $\rho_{1}=0$ ), where $x^{0} \in \mathbb{R}^{N}$ is such that $u\left(x^{0}\right)=\max _{x \in \Omega} u(x)>0$. This actually means that, when $\Omega$ is a ball and $G=\left\{x_{0}\right\}$, Theorem 5 reduces to the classical symmetry result of Gidas, Ni and Nirenberg ([6], see also [5]). Furthermore, in the same situation $\left(G=\left\{x_{0}\right\}\right)$, when $\Omega$ is arbitrary, we obtain an extension of Serrin's result to non-negative solutions. 
THEOREM 6. - Suppose (q) and (f) hold and let $u \in C^{2}(\bar{\Omega})$ be a solution of the following problem

$$
\begin{cases}Q u+f(u,|\nabla u|)=0 & \text { in } \Omega, \\ u \geqslant 0, u \neq \equiv 0 & \text { in } \Omega, \\ u=0, \frac{\partial u}{\partial n}=\mathrm{const} & \text { on } \partial \Omega .\end{cases}
$$

Then $\Omega$ is a ball with radius $\rho_{2}$, centered at $x^{0}, u$ is radial, and

$$
\frac{d u}{d r}<0 \quad \text { for } r=\left|x-x^{0}\right| \in\left(0, \rho_{2}\right) .
$$

The result which we get by putting $\Omega=\mathbb{R}^{N}$ and $G=\left\{x^{0}\right\}$ in Theorem 5 was recently obtained independently by Serrin and Zou in [14] (see also [8]). In this paper they study the symmetry properties of a larger class of elliptic equations on $\mathbb{R}^{N}$, which includes singular operators like the $p$-Laplacian.

Our theorems rely on the widely used method of "moving planes", introduced by Alexandrov and developed in this setting by Serrin.

Finally, we describe several possible extensions of our theorems.

Remark 1. - We can weaken the hypothesis on the regularity of the solution by adding an extra assumption on the shape of the domain $\Omega \backslash G$. All our theorems remain true for weak solutions in $C^{1}(\overline{\Omega \backslash G})$ (as in [14]), provided $\Omega$ and $G$ are such that the critical positions $\lambda_{\star}$ and $\lambda_{\Omega}$ in the moving planes method are always attained when internal tangency occurs (see Section 2 for definitions of these). In particular, this assumption is satisfied for symmetric domains.

If we want to consider $C^{1}$-weak solutions, we need also to suppose that the function $f$ vanishes at $u=0$. Note that this hypothesis is a consequence of the existence of a $C^{2}$-solution.

Remark 2. - After this work was completed the author learned of a recent paper on the strong maximum principle by Pucci, Serrin and Zou [9], where, extending earlier results by Vasquez [16], they establish essentially optimal conditions on the function $f$ under which a non-negative solution of $Q u+f(u,|\nabla u|)=0$ is strictly positive everywhere. They consider differential inequalities and singular elliptic operators.

For instance, when $f$ is independent of $|\nabla u|$, one of their results says the strong maximum principle holds provided

$$
\int_{0} \frac{d u}{\sqrt{F(u)}}=\infty,
$$

where $F(u)=\int_{0}^{u} f(s) d s$. It is elementary to check that (10) is satisfied if $f$ is Lipschitz continuous at $u=0$.

It is not difficult to see that condition (f) can be replaced by the hypotheses in [9] which ensure the validity of the strong maximum principle. 


\section{Proofs}

We apply the method of "moving planes", in order to show that for any direction $\gamma \in \mathbb{R}^{N} \backslash\{0\}$ there exists $\lambda=\lambda(\gamma) \in \mathbb{R}$, such that the domain and the solution are symmetric with respect to the hyperplane

$$
T_{\lambda}=\left\{x \in \mathbb{R}^{N} \mid\langle x, \gamma\rangle=\lambda\right\}
$$

here $\langle\cdot, \cdot\rangle$ denotes the scalar product in $\mathbb{R}^{N}$.

We fix $\gamma$, say $\gamma=e_{1}=(1,0, \ldots, 0)$. For any $x=\left(x_{1}, \ldots, x_{N}\right) \in \mathbb{R}^{N}$ we put $x=\left(x_{1}, x^{\prime}\right) \in \mathbb{R} \times \mathbb{R}^{N-1}$ and denote by $B_{\delta}(x)$ the open ball with radius $\delta$ centered at $x$. For every $A \subset \mathbb{R}^{N}$ and $\lambda, t \in \mathbb{R}$ we set

$$
\begin{aligned}
D_{\lambda} & =\left\{x \in \mathbb{R}^{N} \mid x_{1}>\lambda\right\} ; \\
A^{\lambda} & =\text { the reflection of } A \text { with respect to } T_{\lambda} ; \\
\Gamma_{t}(A) & =A-t e_{1}=\left\{x \in \mathbb{R}^{N} \mid\left(x_{1}+t, x^{\prime}\right) \in A\right\}, \\
\Gamma(A) & =\bigcup_{t \in \mathbb{R}} \Gamma_{t}(A) .
\end{aligned}
$$

For $i=1, \ldots, k$, we define the quantities

$$
\begin{aligned}
& d_{i}=\inf \left\{\lambda \in \mathbb{R} \mid T_{\mu} \cap \overline{G_{i}}=\emptyset \text { for all } \mu>\lambda\right\} ; \\
& \lambda_{i}=\inf \left\{\lambda \leqslant d_{i} \mid \overline{G_{i}} \cap D_{\mu}\right)^{\mu} \subset G_{i} \text { and }\left\langle n(z), e_{1}\right\rangle>0 \\
& \left.\quad \text { for all } \mu>\lambda \text { and all } z \in T_{\mu} \cap \partial G_{i}\right\} ; \\
& d=\max _{1 \leqslant i \leqslant k} d_{i}, \quad \lambda_{\star}=\max _{1 \leqslant i \leqslant k} \lambda_{i} .
\end{aligned}
$$

In other words, $d_{i}$ is the $x_{1}$-coordinate of the right-hand cap of $G_{i}$. Note that $T_{d_{i}}$ is tangent to $\partial G_{i}$ and that $\lambda_{i}<d_{i}$ (this is well-known, see [2]). For a bounded domain $\Omega$ we denote by $d_{\Omega}$ and $\lambda_{\Omega}$ the corresponding values for $\Omega$.

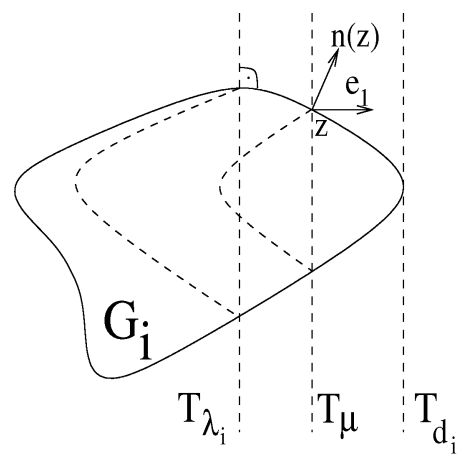

(a)

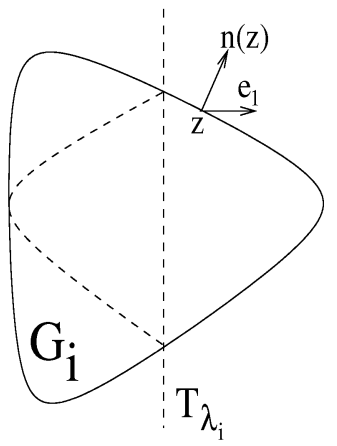

(b)

Fig. 1. Two types of domains $G_{i}$ : the critical position $\lambda_{i}$ is attained at a point of orthogonality (a), or at a point of tangency (b). For all $\mu>\lambda_{i}$ the part of $\overline{G_{i}}$ to the right of $T_{\mu}$ has its reflection inside $G_{i}$ and the outward normal to $\partial G_{i}$ at each point of the boundary of this part makes an acute angle with the direction $e_{1}$. 
We call $\lambda_{i}$ (respectively $\lambda_{\star}$ ) the critical position for $G_{i}$ (respectively $G$ ). We say that $\lambda_{i}$ is attained at a position of internal tangency if $\left(\overline{G_{i}} \cap D_{\lambda_{i}}\right)^{\lambda_{i}} \not \subset G_{i}$ (see Fig. 1). When $\lambda_{i}$ is not attained at a position of internal tangency, we say it is attained at a position of orthogonality.

We set $\Sigma_{\lambda}=\left(D_{\lambda} \cap \Omega\right) \backslash\left(\overline{G^{\lambda}} \cup \bar{G}\right)$ and consider the function

$$
w_{\lambda}(x)=u\left(x^{\lambda}\right)-u(x) .
$$

This function is well defined in $\overline{\Sigma_{\lambda}}$. Our goal is to show that $w_{\lambda} \equiv 0$ in $\Sigma_{\lambda}$ for some $\lambda \in \mathbb{R}$ such that $T_{\lambda}$ is a hyperplane of symmetry for $\Omega$ and $G$.

In the next section we establish some properties of the reflected set $G^{\lambda}$, which we use in Sections 2.2 and 2.3 to prove our theorems.

\subsection{Some reflection properties of the set $G$}

The following easy property of $G$ will permit us to treat this set in most cases like consisting of only one domain.

LEMMA 2.1. - Let $\lambda \geqslant \lambda_{\star}$. For any $i \in\{1, \ldots, k\}$, we have

$$
\overline{G_{i}^{\lambda}} \cap\left(\bigcup_{j \neq i}\left(\overline{G_{j}^{\lambda}} \cup \overline{G_{j}}\right)\right) \cap D_{\lambda}=\emptyset .
$$

Hence any point on $\partial G_{i}^{\lambda} \cap D_{\lambda}$ has a neighbourhood which does not intersect $\bigcup_{j \neq i}\left(\overline{G_{j}^{\lambda}} \cup\right.$ $\left.\overline{G_{j}}\right)$.

Proof. - It is obvious that $\overline{G_{i}} \cap \overline{G_{j}}=\emptyset$ implies $\overline{G_{i}^{\lambda}} \cap \overline{G_{j}^{\lambda}}=\emptyset$. It is also clear that $\lambda \geqslant \lambda_{\star}$ implies, by the definition of $\lambda_{\star}$,

$$
\overline{G_{i}} \cap D_{\lambda} \subseteq \overline{G_{i}^{\lambda}} \cap D_{\lambda},
$$

for all $i \in\{1, \ldots, k\}$. Hence, for any $i \neq j$,

$$
\overline{G_{i}^{\lambda}} \cap\left(\overline{G_{j}^{\lambda}} \cup \overline{G_{j}}\right) \cap D_{\lambda} \subseteq\left(\overline{G_{i}^{\lambda}} \cap \overline{G_{j}^{\lambda}}\right) \cap D_{\lambda}=\emptyset .
$$

We use Lemma 2.1 to obtain a characterisation of the boundary points of the reflected regions $G_{i}^{\lambda}$. It will be crucial in the subsequent discussion.

Let $z \in \partial G_{i}^{\lambda} \cap D_{\lambda}$ be such that $\Gamma_{t}(z)$ belongs to $G_{i}^{\lambda}$ for small positive values of $t$. We define the quantities

$$
\begin{aligned}
\bar{t}=\bar{t}(z) & :=\min \left\{t>0 \mid \Gamma_{t}(z) \in \partial G_{i}^{\lambda}\right\} ; \\
\underline{t}=\underline{t}(z) & :=\min \left\{t>0 \mid \Gamma_{t}(z) \in \partial G_{i}^{\lambda} \cup \partial G_{i}\right\} ; \\
\rho=\rho(z) & :=\operatorname{dist}\left(z, T_{\lambda}\right)>0 .
\end{aligned}
$$

It is clear that $0<\underline{t} \leqslant \bar{t}<\infty$, since $G_{i}$ is smooth and bounded.

Lemma 2.2. - If $\lambda \geqslant \lambda_{\star}$, then any $z \in \partial G_{i}^{\lambda} \cap D_{\lambda}, i=1, \ldots, k$, has one of the following properties (exclusively) 


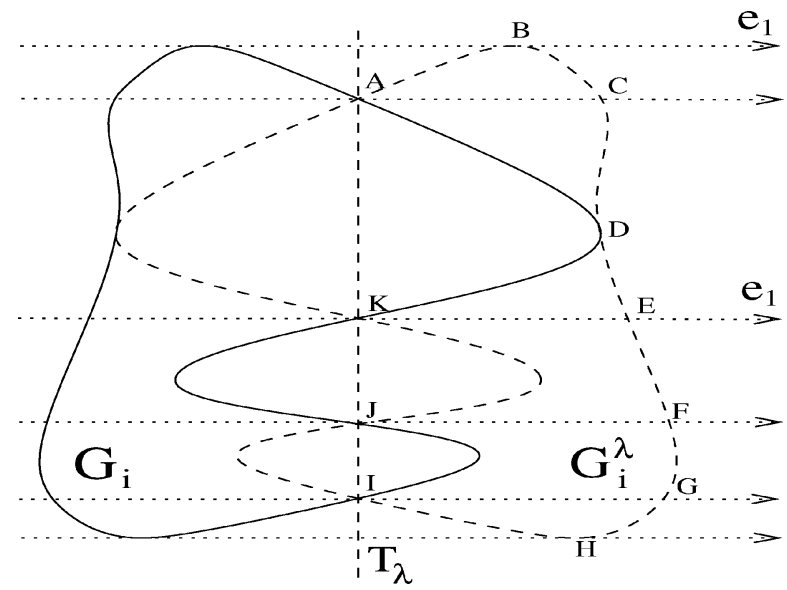

Fig. 2. Four types of points on $\partial G_{i}^{\lambda} \cap D_{\lambda}$ : the $\operatorname{arcs}(\mathrm{A}, \mathrm{B}],[\mathrm{H}, \mathrm{I}),(\mathrm{J}, \mathrm{K})$ are of type $(\mathrm{I})$, the arcs $(\mathrm{B}, \mathrm{C}],[\mathrm{E}, \mathrm{F}],[\mathrm{G}, \mathrm{H})$ are of type $(\mathrm{II})$, the $\operatorname{arcs}(\mathrm{C}, \mathrm{D}),(\mathrm{D}, \mathrm{E}),(\mathrm{F}, \mathrm{G})$ are of type $(\mathrm{III})$, and the point $\mathrm{D}$ is of type (IV).

(I) $\Gamma_{t}(z) \in \Sigma_{\lambda}$ for small positive values of $t$ or there exists a sequence $t_{m} \searrow 0$ such that $\Gamma_{t_{m}}(z) \in \partial G_{i}^{\lambda} \cap D_{\lambda}$;

(II) $\left.0<\underline{t}(z) \leqslant \rho_{(} z\right)$, the open segment $\left(\Gamma_{\underline{t}}(z), z\right)$ belongs to $G_{i}^{\lambda}$, and $\Gamma_{\underline{t}}(z) \in \partial G_{i}^{\lambda}$;

(III) $\left.0<\underline{t}(z)<\rho_{(} z\right)$, the open segment $\left(\bar{\Gamma}_{\underline{t}}(z), z\right)$ belongs to $G_{i}^{\lambda}$, and $\bar{\Gamma}_{\underline{t}}(z) \in \partial G_{i}$;

(IV) $\lambda=\lambda_{\star}$ and $z \in \partial G_{i}^{\lambda} \cap \partial G_{i}$ (the symmetry case).

The four cases of Lemma 2.2 are shown on Fig. 2. In this way we obtain four types of points on $\partial G_{i}^{\lambda} \cap D_{\lambda}$.

Proof of Lemma 2.2. - Fix a point $z \in \partial G_{i}^{\lambda} \cap D_{\lambda}$ and suppose (I) does not hold for $z$. We are going to show that $z$ satisfies one of the other three alternatives.

Since (I) is false for $z$, Lemma 2.1 implies that $\Gamma_{t}(z) \in G_{i}^{\lambda}$ for small positive values of $t$, so that $\bar{t}(z)$ and $\underline{t}(z)$ are well-defined. First we observe that

$$
\bar{t}(z) \leqslant 2 \rho(z) .
$$

Indeed, if this is not true, we obtain a contradiction with the fact that $\lambda \geqslant \lambda_{\star}$. In order to write this rigorously we note that

$$
\left\{\Gamma_{\bar{t}}(z)^{\lambda}\right\}^{\lambda+\frac{1}{2}(\bar{t}-2 \rho)}=z^{\lambda} .
$$

Since $z^{\lambda}$ and $\Gamma_{\bar{t}}(z)^{\lambda}$ are two points on $\partial G_{i}$, this implies that we have internal tangency for $G_{i}$ to the right of or at position $\lambda+\frac{1}{2}(\bar{t}-2 \rho)$. Consequently, in case $\bar{t}>2 \rho$,

$$
\lambda_{\star} \geqslant \lambda_{i} \geqslant \lambda+\frac{1}{2}(\bar{t}-2 \rho)>\lambda
$$

which is a contradiction. 
If $\bar{t}(z)=2 \rho(z)$, then it is obvious that $z^{\lambda}=\Gamma_{\bar{t}}(z) \in \partial G_{i}^{\lambda}$, so

$$
z \in \partial G_{i}^{\lambda} \cap \partial G_{i}
$$

Since in this situation we have tangency to the right of or at position $\lambda$, we deduce $\lambda=\lambda_{\star}$, that is, we are in case (IV).

The last case to consider is $\bar{t}(z)<2 \rho(z)$. We claim that in this case $\underline{t}(z) \leqslant \rho(z)$, that is, $\Gamma_{\underline{t}}(z) \in \overline{D_{\lambda}}$. If $\bar{t}(z) \leqslant \rho(z)$, this is obvious. If

$$
\rho(z)<\bar{t}(z)<2 \rho(z)
$$

then the point $\Gamma_{\bar{t}}(z)^{\lambda} \in \partial G_{i}$ is to the left of $z$ and to the right of $T_{\lambda}$; we infer

$$
\underline{t}(z) \leqslant \operatorname{dist}\left(z, \Gamma_{\bar{t}}(z)^{\lambda}\right)<\rho(z) .
$$

It follows from Lemma 2.1 that if $\underline{t}(z) \leqslant \rho(z)$, then $\underline{t}(z)$ does not change in case we replace $G_{i}$ by $G$ in (11). We infer from this fact that the open segment $\left(\Gamma_{\underline{t}}(z), z\right)$ belongs to $G_{i}^{\lambda}$.

Finally, it is clear that we have either case (II) or case (III), depending on whether the point at which we reach $\underline{t}(z)$ is on $\partial G_{i}^{\lambda}$ or on $\partial G_{i}$.

\subsection{The case $\Omega=\mathbb{R}^{\mathrm{N}}$}

We first give the general plan of the proof of Theorem 2. We use a hyperplane perpendicular to $e_{1}$ and say it has reached a position $\lambda$ provided $w_{\mu} \geqslant 0$ in $\Sigma_{\mu}$ for all $\mu \geqslant \lambda$. The hyperplane "starts" at $\lambda=+\infty$ and "moves" as $\lambda$ decreases. In the initializing step of our proof we show that this process can begin, that is, $w_{\lambda} \geqslant 0$ in $\Sigma_{\lambda}$ for sufficiently large $\lambda$. Next we observe that if the moving plane has reached a position $\lambda$, then the solution $u$ is strictly decreasing - in the $x_{1}$-direction - in the region to the right of the plane $T_{\lambda}$. By using this fact we show that the moving plane reaches position $d$, which permits us to prove that, in a neighbourhood of $\partial G$, the solution $u$ is strictly decreasing in the direction of the outward normal to $\partial G$. Then, having already all the necessary information on the solution, we can show that the moving plane reaches the critical position $\lambda_{\star}$. A device due to Serrin permits us to prove that $w_{\lambda_{\star}} \equiv 0$ in a connected component $Z$ of $\Sigma_{\lambda_{\star}}$. We conclude by showing that all points on $\partial Z \backslash T_{\lambda_{\star}}$ are of the symmetry type (IV) (see Lemma 2.2), and by using a topological argument due to Fraenkel.

We divide the proof of Theorem 2 into ten steps.

STEP 1. - There exists $\bar{\lambda} \in \mathbb{R}$ such that $w_{\lambda} \geqslant 0$ in $\Sigma_{\lambda}$, for all $\lambda \geqslant \bar{\lambda}$.

Proof. - In order to simplify the presentation, we first suppose that we are in the situation of Theorem 1, that is, hypotheses (q) and (f) are replaced by

$\left(\mathrm{q}_{1}\right) \quad Q=\Delta$

$\left(\mathrm{f}_{1}\right) \quad f(u, p)$ does not depend on $p, f \in C^{1}([0, \infty))$ and $f^{\prime}(0)<0$. 
Since $u$ tends to zero at infinity we can take $\tilde{\lambda} \in \mathbb{R}$ such that $\tilde{\lambda}>d$ and

$$
u(x)<\frac{1}{2} \min _{1 \leqslant i \leqslant k} a_{i} \quad \text { for }|x|>\tilde{\lambda},
$$

so that $w_{\lambda}>\frac{a_{i}}{2}>0$ on $\partial G_{i}^{\lambda}$, for all $i$ and all $\lambda \geqslant \tilde{\lambda}$.

Now we can proceed in a standard way. Suppose the claim in Step 1 is false, that is, there exists a sequence $\left\{\lambda_{m}\right\}_{m=1}^{\infty}$ such that

$$
\lim _{m \rightarrow \infty} \lambda_{m}=\infty, \quad \lambda_{m} \geqslant \tilde{\lambda},
$$

and $w_{\lambda_{m}}$ takes negative values in $\Sigma_{\lambda_{m}}$. Since $w_{\lambda}$ is zero on $T_{\lambda}$ and tends to zero at infinity for a fixed $\lambda$, we see that $w_{\lambda_{m}}$ attains its negative minimum inside $\Sigma_{\lambda_{m}}$, say at a point $x^{(m)}$. Then

$$
\nabla w_{\lambda_{m}}\left(x^{(m)}\right)=0 \quad \text { and } \quad \Delta w_{\lambda_{m}}\left(x^{(m)}\right) \geqslant 0 .
$$

On the other hand, it is clear that the function $v_{\lambda}(x)=u\left(x^{\lambda}\right)$ satisfies in $\Sigma_{\lambda}$ the same equation as $u$. By substracting the two equations we see that $w_{\lambda}$ satisfies a linear equation of the type

$$
\Delta w_{\lambda}+b_{\lambda}(x) w_{\lambda}=0 \quad \text { in } \Sigma_{\lambda}
$$

for all $\lambda \in \mathbb{R}$, where $b_{\lambda}(x)=f^{\prime}(c(\lambda, x))$, with

$$
c(\lambda, x) \in\left[\min \left\{u\left(x^{\lambda}\right), u(x)\right\}, \max \left\{u\left(x^{\lambda}\right), u(x)\right\}\right] .
$$

Since $w_{\lambda_{m}}\left(x^{(m)}\right)<0$, we see that $0 \leqslant c\left(\lambda_{m}, x^{(m)}\right) \leqslant u\left(x^{(m)}\right)$ and therefore $\lim _{m \rightarrow \infty} c\left(\lambda_{m}\right.$, $\left.x^{(m)}\right)=0$. It follows that $b_{\lambda_{m}}\left(x^{(m)}\right)$ is strictly negative for large $m$. Hence

$$
0 \leqslant \Delta w_{\lambda_{m}}\left(x^{(m)}\right)=-b_{\lambda_{m}}\left(x^{(m)}\right) w_{\lambda_{m}}\left(x^{(m)}\right)<0,
$$

which is a contradiction.

The way to extend this argument to the general case is well-known. We sketch it here, for the sake of completeness. After substracting the two equations for $u$ and $v_{\lambda}$ and doing some standard computations, we obtain a linear strictly elliptic equation with bounded coefficients, in the form

$$
\partial_{i}\left(a_{i j}(x) \partial_{j} w_{\lambda}\right)+b_{i}(x) \partial_{i} w_{\lambda}+c(x) w_{\lambda}=0,
$$

where $c(x) \leqslant 0$, provided the functions $u(x)$ and $v_{\lambda}(x)$ are sufficiently small. Then we can use the weak maximum principle, as in [7], to conclude that $w_{\lambda} \geqslant 0$ in $\Sigma_{\lambda}$, for $\lambda$ sufficiently large.

Step 1 shows that the number

$$
\lambda_{0}=\inf \left\{\lambda \in \mathbb{R} \mid w_{\mu} \geqslant 0 \text { in } \Sigma_{\mu} \text { for all } \mu>\lambda\right\}
$$

is well defined. It is obvious that $\lambda_{0}$ is finite. Notice that, by continuity, $w_{\lambda_{0}} \geqslant 0$ in $\Sigma_{\lambda_{0}}$. 
STEP 2. - We have

$$
\frac{\partial u}{\partial x_{1}}<0
$$

in the set $\left\{x \in \mathbb{R}^{N} \mid x_{1}>\max \left\{\lambda_{0}, d\right\}\right\}$.

Proof. - Fix $\mu>\max \left\{\lambda_{0}, d\right\}$. Notice that for all $\lambda>d$ the set $\Sigma_{\lambda}$ is connected and regular. It follows from the strong maximum principle and Hopf's lemma, applied to (13), that for any $\lambda \in\left[\lambda_{0}, \infty\right) \cap(d, \infty)$, either $w_{\lambda} \equiv 0$ in $\Sigma_{\lambda}$ or $w_{\lambda}>0$ in $\Sigma_{\lambda}$, with $\frac{\partial w_{\lambda}}{\partial v}<0$ on points of $\partial \Sigma_{\lambda}$ at which $w_{\lambda}$ takes value zero (here and in the sequel $v$ denotes the outward normal to $\partial \Sigma_{\lambda}$ ).

If $w_{\mu}>0$ in $\Sigma_{\mu}$ we obtain, using the fact that $w_{\mu}=0$ on $T_{\mu}$,

$$
0>\left.\frac{\partial w_{\mu}}{\partial v}\right|_{T_{\mu}}=-\left.\frac{\partial w_{\mu}}{\partial x_{1}}\right|_{T_{\mu}}=\left.2 \frac{\partial u}{\partial x_{1}}\right|_{T_{\mu}} .
$$

Supose for contradiction that $w_{\mu} \equiv 0$ in $\Sigma_{\mu}$. We distinguish two cases. First, if $w_{\lambda}>0$ in $\Sigma_{\lambda}$ for all $\lambda>\mu$ then, using (14) with $\mu$ replaced by $\lambda$, we see that $\frac{\partial u}{\partial x_{1}}<0$ in $D_{\mu}$. Then we fix a point $\bar{x} \in \Sigma_{\mu}$ such that $\Gamma(\bar{x}) \cap \bar{G}=\emptyset$, and obtain the contradiction

$$
u(\bar{x}) \leqslant u\left(\bar{x}^{\lambda_{0}}\right)=u\left(\left(\bar{x}^{\lambda_{0}}\right)^{\mu}\right)=u\left(\bar{x}_{1}+2\left(\mu-\lambda_{0}\right), \bar{x}^{\prime}\right)<u(\bar{x})
$$

(see Fig. 3).

Second, if $w_{\widetilde{\mu}} \equiv 0$ in $\Sigma_{\widetilde{\mu}}$ for some $\widetilde{\mu}>\mu$, we take a point $\bar{y} \in \partial G$ such that $\Gamma(\bar{y}) \cap G=\emptyset$ and, by using consecutive reflexions with respect to $T_{\mu}$ and $T_{\widetilde{\mu}}$, obtain an unbounded sequence of points at which $u$ takes a fixed positive value - contradiction with (BC).

STEP 3. $-\lambda_{0} \leqslant d$.

Proof. - Suppose for contradiction that $\lambda_{0}>d$. As explained above, either $w_{\lambda_{0}} \equiv 0$ in $\Sigma_{\lambda_{0}}$ or $w_{\lambda_{0}}>0$ in $\Sigma_{\lambda_{0}}$. First assume $w_{\lambda_{0}} \equiv 0$ in $\Sigma_{\lambda_{0}}$. We can always take two points

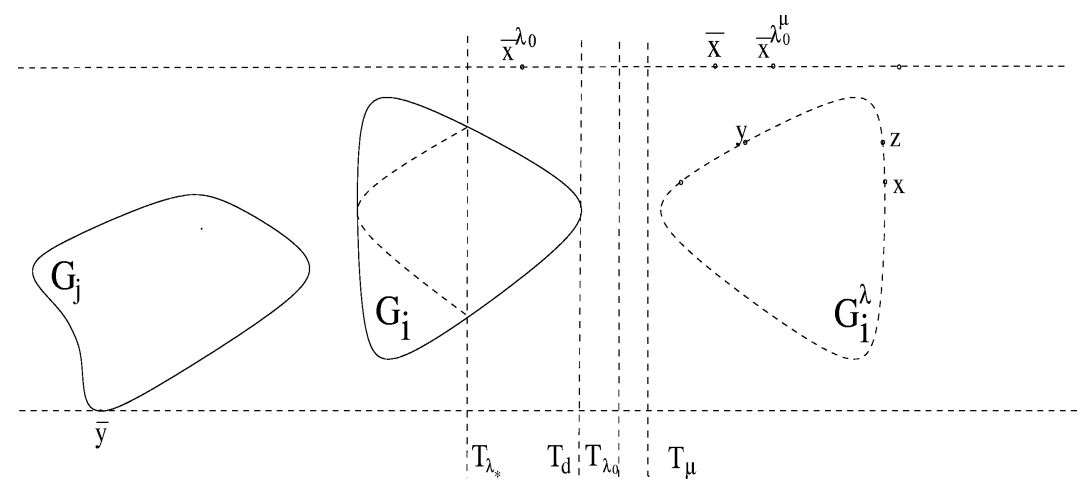

Fig. 3. The contradictions in Steps 2 and 3. 
$y, z \in \partial G_{1}^{\lambda_{0}}$ such that $y_{1}<z_{1}$ and $y^{\prime}=z^{\prime}$. Then, by Step $2, u(y)>u(z)$. However, we get

$$
u(y)=u\left(y^{\lambda_{0}}\right)=a_{1}=u\left(z^{\lambda_{0}}\right)=u(z),
$$

because $w_{\lambda_{0}} \equiv 0$.

Next, suppose $w_{\lambda_{0}}>0$ in $\Sigma_{\lambda_{0}}$, with $\frac{\partial w_{\lambda_{0}}}{\partial v}<0$ on points of $\partial \Sigma_{\lambda_{0}}$ where $w_{\lambda_{0}}=0$. By using the minimal choice of $\lambda_{0}$ we can find a sequence $\left\{\lambda_{m}\right\}_{m=1}^{\infty}$ such that $\lim _{m \rightarrow \infty} \lambda_{m}=$ $\lambda_{0}, \lambda_{m}<\lambda_{0}$, and $w_{\lambda_{m}}$ takes negative values in $\Sigma_{\lambda_{m}}$. Let $x^{(m)} \in \overline{\Sigma_{\lambda_{m}}}$ be such that

$$
w_{\lambda_{m}}\left(x^{(m)}\right)=\min _{x \in \overline{\Sigma_{\lambda_{m}}}} w_{\lambda_{m}}(x)<0 .
$$

Using the assumption $\lambda_{0}>d$, we fix $m_{0}$ such that

$$
\operatorname{dist}\left(\overline{G^{\lambda_{m}}}, T_{\lambda_{0}}\right) \geqslant \frac{1}{2} \operatorname{dist}\left(\overline{G^{\lambda_{0}}}, T_{\lambda_{0}}\right)>0,
$$

for $m \geqslant m_{0}$.

We distinguish two cases.

Case 1. $x^{(m)} \in \operatorname{int} \Sigma_{\lambda_{m}}$, for all $m \geqslant m_{0}$.

In this case a contradiction is obtained in a standard way. As in Step 1 we see that $\Delta w_{\lambda_{m}}\left(x^{(m)}\right) \geqslant 0$ and $\nabla w_{\lambda_{m}}\left(x^{(m)}\right)=0$. If $\left\{x^{(m)}\right\}$ tends to infinity we obtain a contradiction exactly as in Step 1. If a subsequence of $\left\{x^{(m)}\right\}$ converges to a point $x^{0}$, then by passing to the limit we obtain $x^{0} \in \overline{\Sigma_{\lambda_{0}}}, w_{\lambda_{0}}\left(x^{0}\right) \leqslant 0$ and $\nabla w_{\lambda_{0}}\left(x^{0}\right)=0$. This means that $x^{0} \in \partial \Sigma_{\lambda_{0}}$ and $w_{\lambda_{0}}\left(x^{0}\right)=0$, so Hopf's lemma implies $\nabla u\left(x^{0}\right) \neq 0$, a contradiction.

Case 2. $x^{(m)} \in \partial \Sigma_{\lambda_{m}}$, for some $m \geqslant m_{0}$.

We drop the super(sub-)script $m$, for simplicity. Since $w_{\lambda}=0$ on $T_{\lambda}$, we see that $x \in \partial G_{i}^{\lambda}$, for some $i \in\{1, \ldots, k\}$. We infer from (15) that $\Gamma_{t}(x)$ belongs to $D_{\lambda_{0}}$, for small $t>0$.

We apply Lemma 2.2, which says $x$ has one of the four properties described in the statement of this lemma. Here $\lambda>d>\lambda_{\star}$, hence $x$ cannot be of type (IV). It cannot be of type (III) either, since $\bar{G} \subset \mathbb{R}^{N} \backslash \overline{D_{\lambda}}$. We are going to obtain a contradiction in the remaining two cases.

First, suppose $x$ is of type (I). If $\Gamma_{t}(x)$ belongs to $\Sigma_{\lambda}$ for small $t>0$ we consider the directional derivative $\frac{\partial w_{\lambda}}{\partial\left(-e_{1}\right)}(x)$. By the minimal choice of $x$ this derivative has to be non-negative. On the other hand, it is easy to see that

$$
\frac{\partial w_{\lambda}}{\partial\left(-e_{1}\right)}(x)=\frac{\partial u}{\partial x_{1}}(x)+\frac{\partial u}{\partial x_{1}}\left(x^{\lambda}\right)<\frac{\partial u}{\partial x_{1}}\left(x^{\lambda}\right),
$$

by Step 2 .

We are going to show that $\frac{\partial u}{\partial x_{1}}\left(x^{\lambda}\right)$ is non-positive. The fact that $\Gamma_{t}(x) \in \Sigma_{\lambda}$ for small $t>0$ implies $\left\langle n\left(x^{\lambda}\right), e_{1}\right\rangle \geqslant 0$ (it is a standard fact that a direction which makes an acute angle with the inward normal to the boundary of a smooth domain enters the domain). It is clear that $u \equiv$ const on $\partial G_{i}$ implies

$$
\left.\frac{\partial u}{\partial \xi_{j}}\right|_{\partial G_{i}} \equiv 0, \quad j=1, \ldots, N-1,
$$


for any orthonormal basis $\xi_{1}, \ldots, \xi_{N-1}$ in the tangent plane to $\partial G_{i}$. Hence, by (BC),

$$
\frac{\partial u}{\partial x_{1}}\left(x^{\lambda}\right)=\left\langle n\left(x^{\lambda}\right), e_{1}\right\rangle \frac{\partial u}{\partial n}\left(x^{\lambda}\right) \leqslant 0,
$$

which leads to a contradiction.

Next, still in case (I), suppose that there exists a sequence of positive numbers $t_{m} \rightarrow 0$, such that $x^{(m)}:=\Gamma_{t_{m}}(x) \in \partial G_{i}^{\lambda}$. We have

$$
w_{\lambda}\left(x^{(m)}\right) \geqslant w_{\lambda}(x)
$$

by the minimal choice of $x$. On the other hand, Step 2 together with $t_{m}>0$ implies $u\left(x^{(m)}\right)>u(x)$. Hence

$$
w_{\lambda}\left(x^{(m)}\right)=a_{i}-u\left(x^{(m)}\right)<a_{i}-u(x)=w_{\lambda}(x)
$$

a contradiction.

Finally, if $x$ is of type (II), we see that $\Gamma_{\underline{t}}(x) \in \partial G_{i}^{\lambda} \cap D_{\lambda_{0}}$ (since $\overline{G^{\lambda}} \subset D_{\lambda_{0}}$ ), where $\underline{t}=\underline{t}(x)$ is the number defined in Section 2. 1 . Hence, by Step 2,

$$
w_{\lambda}\left(\Gamma_{\underline{t}}(x)\right)=a_{i}-u\left(\Gamma_{\underline{t}}(x)\right)<a_{i}-u(x)=w_{\lambda}(x),
$$

which contradicts the minimal choice of $x$.

Remark 3. - We can now prove Theorem 5 for $G=\left\{x^{0}\right\}$, where $x^{0} \in \mathbb{R}^{N}$ is such that $u\left(x^{0}\right)=\max _{x \in \mathbb{R}^{N}} u(x)>0$. By Step $3, \lambda_{0} \leqslant x_{1}^{0}$ and $w_{\lambda_{0}} \geqslant 0$ in $\Sigma_{\lambda_{0}}$. By taking a moving plane coming from the left we get $w_{x_{1}^{0}} \equiv 0$ in $\Sigma_{x_{1}^{0}}$. To be more precise, by "a plane coming from the left" we mean the same process of moving a hyperplane $T_{\lambda}$ which starts at $\lambda=-\infty$ and moves as $\lambda$ increases. The region $\Sigma_{\lambda}$ would then be defined to the left of $T_{\lambda}$.

STEP 4. - For any $z \in \partial G$ and any unit vector $\eta$, for which $\langle\eta, n(z)\rangle>0$, we can find a sufficiently small ball $B_{\delta}(z)$ such that

$$
\frac{\partial u}{\partial \eta}(\zeta)<0 \quad \text { for all } \zeta \in B_{\delta}(z) \backslash \bar{G}
$$

This statement was already used by Reichel, who established it under condition (r). Since we do not assume this stronger condition, we have to provide a different proof.

The proof of Step 4 is the only place in Section 2.2 where we have to be careful about the fact that there might be more than one domain $G_{i}$. We postpone this proof for the time being.

STEP 5. $-w_{\lambda}>0$ in $\Sigma_{\lambda}$, for any $\lambda \in\left[\lambda_{0}, \infty\right) \cap\left(\lambda_{\star}, \infty\right)$.

Proof. - In view of Steps 2 and 3 we can restrict ourselves to the case $\lambda \leqslant d$. By the strong maximum principle, all we have to exclude is $w_{\lambda} \equiv 0$ in a connected component $Z$ of $\Sigma_{\lambda}$. Suppose for contradiction we are in this situation. 


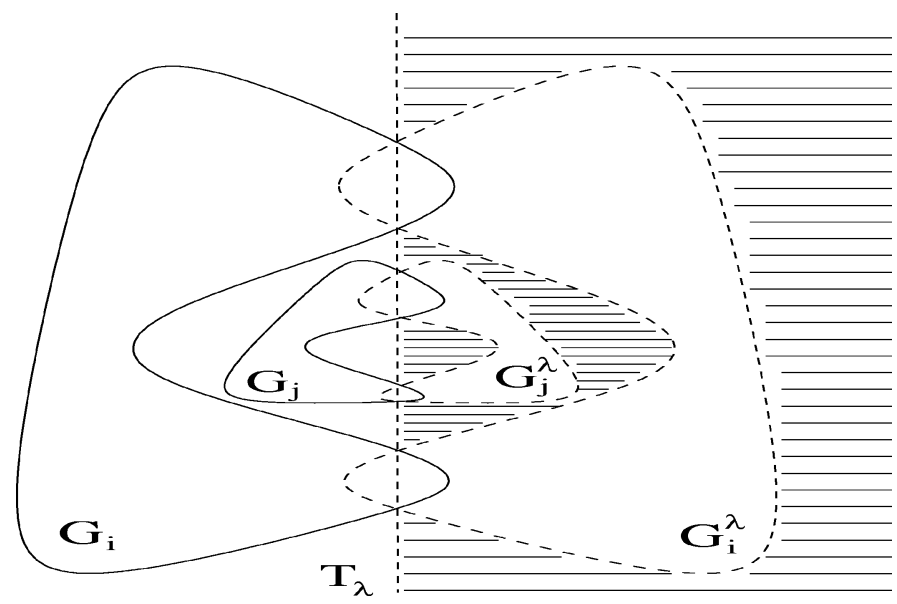

Fig. 4. The shaded regions are the connected components of $\Sigma_{\lambda}$.

First we observe that any connected component $Y$ of $\Sigma_{\lambda}$ satisfies

$$
\operatorname{dist}\left(\partial Y \cap D_{\lambda}, T_{\lambda}\right)=0
$$

(see Fig. 4). Indeed, $Y$ is connected, so $Y^{\lambda}$ is also connected and hence $Y^{\lambda}$ is a connected component of $\left(\mathbb{R}^{N} \backslash \bar{G}\right) \backslash \overline{D_{\lambda}}$. Since $\mathbb{R}^{N} \backslash \bar{G}$ is itself connected, we see that either $Y^{\lambda}$ contains a left neighbourhood of $T_{\lambda}$ or

$$
\operatorname{dist}\left(\partial Y^{\lambda} \backslash \overline{D_{\lambda}}, T_{\lambda}\right)=0
$$

The first alternative is impossible because of the fact that $T_{\lambda}$ touches at least one of the domains $G_{i}$ (recall that $\lambda \leqslant d$ ). By reflection (17) implies (16).

Now (16) permits us to take a sequence $\left\{z^{(m)}\right\}_{m=1}^{\infty} \subset \partial Z \cap D_{\lambda}$, which converges to a point $z^{0} \in T_{\lambda} \cap \partial Z \cap \partial G$. Since $\lambda>\lambda_{\star}$, we have $\left\langle n\left(z^{(m)^{\lambda}}\right), e_{1}\right\rangle>0$ for sufficiently large $m$, and the open segment between $z^{(m)^{\lambda}}$ and $z^{(m)}$ belongs to $B_{\delta}\left(z^{0}\right) \backslash \bar{G}$, where $\delta$ is chosen as in Step 4. Then, by Step 4, $u$ decreases strictly from $z^{(m)^{\lambda}}$ to $z^{(m)}$, which yields $w_{\lambda}\left(z^{(m)}\right)>0$, a contradiction with the fact that $w_{\lambda} \equiv 0$ in $Z$. This last argument was used by Reichel in [10].

STEP 6. - We have

$$
\frac{\partial u}{\partial x_{1}}<0 \quad \text { in } D_{\lambda^{\star}} \backslash \bar{G}
$$

where $\lambda^{\star}=\max \left\{\lambda_{0}, \lambda_{\star}\right\}$.

Proof. - By proceeding as in Step 2, we see that this is a direct consequence of Step 5 and Hopf's lemma.

STEP 7. $-\lambda_{0} \leqslant \lambda_{\star}$. 
Proof. - Suppose $\lambda_{0}>\lambda_{\star}$. By Step 5 we know that $w_{\lambda_{0}}>0$ in $\Sigma_{\lambda_{0}}$. Proceeding as in Step 3, we find two sequences $\left\{\lambda_{m}\right\}_{m=1}^{\infty},\left\{x^{(m)}\right\}_{m=1}^{\infty}$, such that

$$
\lim _{m \rightarrow \infty} \lambda_{m}=\lambda_{0}, \quad \lambda_{\star}<\lambda_{m}<\lambda_{0}, \quad x^{(m)} \in \overline{\Sigma_{\lambda_{m}}} \backslash T_{\lambda_{m}}
$$

and $w_{\lambda_{m}}$ attains its negative minimum in $\overline{\Sigma_{\lambda_{m}}}$ at $x^{(m)}$.

A number of different situations may arise. We are going to obtain a contradiction in each of them.

Case 1. There is a subsequence of $\left\{x^{(m)}\right\}$, such that $x^{(m)} \in$ int $\Sigma_{\lambda_{m}}$.

If $\lim _{m \rightarrow \infty}\left|x^{(m)}\right|=\infty$ we obtain a contradiction as in Step 1. If a subsequence of $\left\{x^{(m)}\right\}$ converges to a point $x^{0}$ which belongs to the regular part of $\partial \Sigma_{\lambda_{0}}$, we obtain a contradiction as in Case 1 of Step 3. If $x^{0}$ belongs to the singular part of $\partial \Sigma_{\lambda_{0}}$, we have $x^{0} \in T_{\lambda_{0}} \cap \partial G$, so that the argument we used at the end of the proof of Step 5 implies $w_{\lambda_{m}}\left(x^{(m)}\right)>0$, for $m$ sufficiently large.

Case 2. There is a subsequence of $\left\{x^{(m)}\right\}$, such that $x^{(m)} \in \partial \Sigma_{\lambda_{m}}$.

Notice that in this case $\left\{x^{(m)}\right.$ is bounded, since $\partial \Sigma_{\lambda_{m}} \backslash T_{\lambda_{m}}$ is a bounded set. Fix $i$ such that (along a subsequence) $x^{(m)} \in \partial G_{i}^{\lambda_{m}}$. We shall apply Lemma 2.2 to $x^{(m)}$ and reach a contradiction in all its four cases, with the help of Step 6. However, Step 6 gives information on the behaviour of the solution only to the right of $T_{\lambda_{0}}$, so we have first to exclude the case when $\left\{x^{(m)}\right\} \subset \partial \Sigma_{\lambda_{m}} \backslash D_{\lambda_{0}}$ (see Fig. 5). If such a subsequence exists, then $\lambda_{m} \rightarrow \lambda_{0}$ implies (along a subsequence) $x^{(m)} \rightarrow x^{0} \in T_{\lambda_{0}} \cap \partial G_{i}$. Then the argument we used at the end of the proof of Step 5 leads to a contradiction.

Therefore we can assume that $x^{(m)} \in \partial G_{i}^{\lambda_{m}} \cap D_{\lambda_{0}}$. We apply Lemma 2.2 to $x^{(m)}$. If, for some $m$, the point $x^{(m)}$ is of type (I) we obtain a contradiction exactly as in Step 3 . Points of type (IV) are also excluded, since $\lambda_{m}>\lambda_{\star}$.

It remains to reach a contradiction in case $x^{(m)}$ is of type (II) or (III) for all $m$. Let $\underline{t}=\underline{t}(m)$ be the quantity defined in Section 2.1 and let $\underline{x}^{(m)}:=\Gamma_{\underline{t}}\left(x^{(m)}\right)$. We treat separately the cases when $\underline{x}^{(m)}$ belongs or not to $D_{\lambda_{0}}$ (note that by Lemma 2.2 we only know that $\underline{x}^{(m)} \in \overline{D_{\lambda_{m}}}$, see Fig. 5).

Case 2.1. $\underline{x}^{(m)} \in D_{\lambda_{0}}$ for some $m \in \mathbb{N}$.

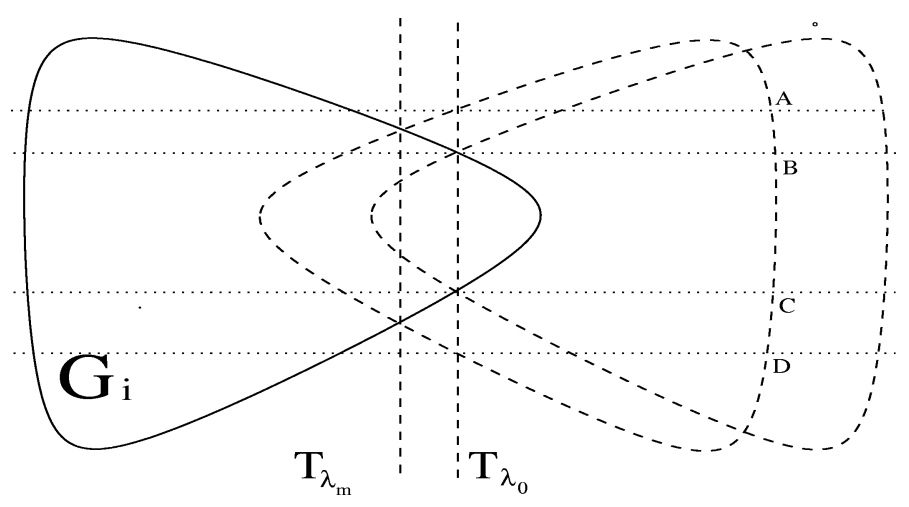

Fig. 5. The different situations in Case 2. For instance, the points on the arcs $[A, B]$ and $[C, D]$ give rise to Case 2.2. 
First suppose $x^{(m)}$ is of type (II), that is, $\underline{x}^{(m)} \in \partial G_{i}^{\lambda_{m}} \cap D_{\lambda_{0}}$. Then we obtain a contradiction with the minimal choice of $x^{(m)}$, exactly as in Step 3 .

Second suppose $x^{(m)}$ is of type (III), that is, $\underline{x}^{(m)} \in \partial G_{i} \cap D_{\lambda_{0}}$. Then $\left\langle n\left(\underline{x}^{(m)}\right), e_{1}\right\rangle>0$ (since $\lambda_{0}>\lambda_{\star}$ ). Therefore $u$ decreases strictly from $\underline{x}^{(m)}$ to $x^{(m)}$ (indeed, by Step 4 it starts from $\underline{x}^{(m)}$ by decreasing and continues decreasing, by Step 6). Hence $u\left(x^{(m)}\right)<$ $u\left(\underline{x}^{(m)}\right)=a_{i}$, and

$$
w_{\lambda_{m}}\left(x^{(m)}\right)=a_{i}-u\left(x^{(m)}\right)>0,
$$

a contradiction (recall that $w_{\lambda_{m}}$ is negative at $x^{(m)}$ ).

Case 2.2. $\underline{x}^{(m)} \in \overline{D_{\lambda_{m}}} \backslash D_{\lambda_{0}}$ for all $m \in \mathbb{N}$.

Since $\underline{x}^{(m)} \in \partial G_{i} \cup \partial G_{i}^{\lambda_{m}}$, we see that $\left\{x^{(m)}\right\}$ is bounded. So we can extract a subsequence $\underline{x}^{(m)} \rightarrow \underline{x}^{0} \in \partial G_{i} \cap T_{\lambda_{0}}=\partial G_{i}^{\lambda_{0}} \cap T_{\lambda_{0}}$. Then for $m$ sufficiently large the segment between $\underline{x}^{m}$ and its orthogonal projection on $T_{\lambda_{0}}$ belongs to $B_{\delta}\left(\underline{x}^{0}\right)$. Step 4 implies that $u$ decreases on that segment. By using Step 6 to the right of $T_{\lambda_{0}}$, we see that $u$ decreases on the whole segment $\left(\underline{x}^{(m)}, x^{(m)}\right)$. Then we obtain a contradiction exactly as in Case 2.1, for both types of points.

STEP 8. $-w_{\lambda_{\star}} \equiv 0$ in at least one connected component of $\Sigma_{\lambda_{\star}}$.

Proof. - By Step 7, $w_{\lambda_{\star}} \geqslant 0$ in $\Sigma_{\lambda_{\star}}$. The following argument was carried out in [11]. We outline the proof for completeness.

If there exists $z^{0} \in \partial G \cap T_{\lambda_{\star}}$ such that $\left\langle n\left(z^{0}\right), e_{1}\right\rangle=0$, one can show that all first and second order derivatives of $w_{\lambda_{\star}}$ vanish at $z^{0}$ (see [11], pp. 389-391 for detailed computations). Then Serrin's corner lemma (see [13]) immediately implies that $w_{\lambda_{\star}} \equiv 0$ in the connected component of $\Sigma_{\lambda_{\star}}$ whose boundary contains $z^{0}$.

If no such $z^{0}$ exists, by the definition of $\lambda_{\star}$ we can find $z_{1} \in \partial G \cap D_{\lambda_{\star}}$ such that $z_{1}^{\lambda_{\star}} \in \partial G$ and $\left(\partial\left(G \cap D_{\lambda_{\star}}\right)\right)^{\lambda_{\star}}$ is internally tangent to $\partial G$ at $z_{1}^{\lambda_{\star}}$. By (BC), $w_{\lambda_{\star}}\left(z_{1}\right)=0$ and $\frac{\partial w_{\lambda_{\star}}}{\partial v}\left(z_{1}\right)=0$, so the claim of Step 8 follows from Hopf's lemma.

STEP 9. - Let $Z$ be a connected component of $\Sigma_{\lambda_{\star}}$ such that $w_{\lambda_{\star}} \equiv 0$ in $Z$. Then

$$
\partial Z \backslash T_{\lambda_{\star}} \subset \partial G
$$

Proof. - We shall once more make use of Lemma 2.2. Another way of stating Step 9 is to say that all points on $\partial Z \backslash T_{\lambda_{\star}}$ are of the symmetry type (IV). Suppose for contradiction that there exists a point $z \in \partial Z \backslash T_{\lambda_{\star}}$ (say $z \in \partial G_{i}^{\lambda_{\star}}$ ), which is not of type (IV). We are going to obtain a contradiction in all of the remaining three cases.

The point $z$ is not of type (I), since if it were, $w_{\lambda_{\star}} \equiv 0$ in $Z$ would lead to a contradiction, exactly as in Step 3. If $z$ is of type (II) or (III), we set, as before, $\underline{z}=\Gamma_{\underline{t}(z)}(z) \in \overline{D_{\lambda_{\star}}}$. Then Step 6 implies that $u$ decreases strictly on the segment $(\underline{z}, z)$. In case (II) we get

$$
a_{i}=u\left(\underline{z}^{\lambda_{\star}}\right) \geqslant u(\underline{z})>u(z)=u\left(z^{\lambda_{\star}}\right)=a_{i},
$$

since $w_{\lambda_{\star}} \geqslant 0$ in $\Sigma_{\lambda_{\star}}$ and $w_{\lambda_{\star}}(z)=0$. Analogously, in case (III) we have

$$
a_{i}=u(\underline{z})>u(z)=u\left(z^{\lambda_{\star}}\right)=a_{i} .
$$


Step 9 follows.

STEP 10. - Conclusion.

Proof. - Once we have proved Step 9, the conclusion is obtained via a topological argument, due to Fraenkel and used in this setting by Reichel. Note that under condition (r) the previous Step 9 is obvious and is independent of all other steps.

We sketch the argument for completeness. Set $G^{c}=\mathbb{R}^{N} \backslash G$ and

$$
X=Z \cup Z^{\lambda} \cup\left(\partial Z \cap G^{c}\right) \cup\left(\partial Z^{\lambda} \cap G^{c}\right) .
$$

The set $X$ is symmetric with respect to $T_{\lambda}$, since Step 9 implies

$$
\partial Z \cap G^{c} \subset T_{\lambda} \cap G^{c} .
$$

One may check that $X$ is open in $G^{c}$ and hence $\partial X \subset \partial G$. Then $G^{c} \backslash X=G^{c} \backslash \bar{X}$, which implies that $X=G^{c}$, since $G^{c}$ is connected.

Finally we go back to Step 4.

Proof of Step 4. - We use induction with respect to $k$. First assume $k=1$. If $\alpha_{1}<0$, Step 4 is obvious, by continuity. Hence we can assume $\alpha_{1}=0$, or equivalently $\nabla u \equiv 0$ on $\partial G$. This implies $\left|D^{2} u\right|=\left|\frac{\partial^{2} u}{\partial n^{2}}\right|$ on $\partial G$.

Fix a point $z^{0} \in T_{d} \cap \partial G$, so that

$$
\frac{\partial u}{\partial x_{1}}\left(z^{0}\right)=\frac{\partial u}{\partial n}\left(z^{0}\right)=0 .
$$

Steps 2 and 3, together with the assumption $\lambda_{0} \leqslant d$, imply

$$
\frac{\partial u}{\partial x_{1}}\left(\Gamma_{t}\left(z^{0}\right)\right)<0
$$

for negative $t$. We conclude that

$$
\frac{\partial^{2} u}{\partial n^{2}}\left(z^{0}\right)=\frac{\partial^{2} u}{\partial x_{1}^{2}}\left(z^{0}\right) \leqslant 0 .
$$

On the other hand, it is easy to compute that $u=$ const and $\nabla u=0$ on $\partial G$ imply

$$
\left.\operatorname{div}(g(|\nabla u|) \nabla u)\right|_{\partial G}=\left.g(|\nabla u|) \Delta u\right|_{\partial G}+\left\langle\nabla[g(|\nabla u|)],\left.\nabla u\right|_{\partial G}=\left.g(0) \Delta u\right|_{\partial G}\right.
$$

and

$$
\left.\Delta u\right|_{\partial G}=\left.\frac{\partial^{2} u}{\partial n^{2}}\right|_{\partial G}
$$

Hence

$$
\frac{\partial^{2} u}{\partial n^{2}} \equiv-\frac{f\left(a_{1}, 0\right)}{g(0)}=\mathrm{const} \quad \text { on } \partial G
$$


By $(18), f\left(a_{1}, 0\right) \geqslant 0$. If $f\left(a_{1}, 0\right)>0$, Step 4 follows easily, since

$$
\frac{\partial^{2} u}{\partial \eta^{2}}=\langle\eta, n\rangle^{2} \frac{\partial^{2} u}{\partial n^{2}} \quad \text { on } \partial G
$$

If $f\left(a_{1}, 0\right)=0$, we see that all first and second order derivatives of $u$ vanish on $\partial G$. This implies that the function

$$
\bar{u}(x)= \begin{cases}u(x) & \text { for } x \in \mathbb{R}^{N} \backslash G, \\ a_{1} & \text { for } x \in \bar{G}\end{cases}
$$

belongs to $C^{2}\left(\mathbb{R}^{N}\right)$ and solves the equation

$$
\begin{cases}Q \bar{u}+f(\bar{u},|\nabla \bar{u}|)=0 & \text { in } \mathbb{R}^{N}, \\ \bar{u} \geqslant 0, \bar{u} \neq 0 & \text { in } \mathbb{R}^{N}, \\ \bar{u}(x) \rightarrow 0 & \text { as } x \rightarrow \infty\end{cases}
$$

However, the shape of $\bar{u}$ contradicts the result for equations on $\mathbb{R}^{N}$ that we have already proved (see Remark 3), which says a solution of (19) has a non-zero gradient everywhere, except at one point.

Suppose next that Step 4 is proved for $k-1$ domains $G_{i}$, and consider a problem with $k$ domains. By Steps 5-10, Theorem 2 holds for problems with $k-1$ domains $G_{i}$. Set

$$
I=\left\{i \mid \alpha_{i}<0 \text { or } \frac{\partial^{2} u}{\partial n^{2}}<0 \text { on } \partial G_{i}\right\}
$$

and $J=\{1, \ldots, k\} \backslash I$. Note that the statement of Step 4 is true for $G_{i}$ with $i \in I$.

We claim that $J$ is empty. Suppose this is not the case, and set

$$
\underline{d}=\max _{j \in J} d_{j}
$$

It is clear that Step 5 can be proved for values of $\lambda$ such that $\lambda \geqslant \lambda_{0}$ and $\lambda>\max \left\{\lambda_{\star}, \underline{d}\right\}$. Hence, as in Step 6,

$$
\frac{\partial u}{\partial x_{1}}<0 \quad \text { in } D_{\max \left\{\lambda_{\star}, \underline{d}\right\}} \backslash \bar{G} .
$$

It follows that, if $\lambda_{\star}>\underline{d}$, Steps 7-10 hold with the same proofs, yielding a contradiction. If $\lambda_{\star} \leqslant \underline{d}$ the moving plane reaches at least one domain $G_{j_{0}}$, with $j_{0} \in J$. As in the case $k=1$, this implies $f\left(a_{j_{0}}, 0\right) \geqslant 0$ and $f\left(a_{j_{0}}, 0\right)=0$, by the definition of $J$. Then, defining the function $\bar{u}$ with $a_{1}$ replaced by $a_{j_{0}}$ and $\bar{G}$ replaced by $\overline{G_{j_{0}}}$, we obtain a contradiction with Theorem 2 , in the case when there are $k-1$ domains.

This concludes the proof of Theorem 2 for $\Omega=\mathbb{R}^{N}$.

Proof of Theorem 5. - We now suppose that $G$ is a ball and (8) holds. Observe that the hypotheses of Theorem 5 are sufficient to carry out the first three steps in the above proof. It is easy to see that Step 3 implies $u<a$ in $\mathbb{R}^{N} \backslash G$. Indeed, if there exists 
$x \in \mathbb{R}^{N} \backslash G$ such that $u(x) \geqslant a$, we take moving planes $T_{\lambda(\gamma)}$, for $\gamma=x$, and see that $u$ has to increase strictly on the ray from $x$ to the boundary $\partial G$, which is a contradiction, since $u=a$ on $\partial G$.

The rest of the proof is elementary. We know that $w_{\lambda}>0$ in $\Sigma_{\lambda}$ for $\lambda>\max \left\{\lambda_{0}, \lambda_{\star}\right\}$. To prove $\lambda_{0} \leqslant \lambda_{\star}$, we have only to consider Case 1 in Step 7. It remains the same, since $u<a$ in $\mathbb{R}^{N} \backslash G$ implies Step 4 (see for example [11]). Finally, $w_{\lambda_{\star}} \equiv 0$ is obtained with the help of a moving plane coming from the left.

\subsection{Bounded domains $\Omega$}

We begin with a simple but basic observation, which permits us to treat this case in essentially the same way as the case $\Omega=\mathbb{R}^{N}$.

LEMMA 2.3. - If $w_{\lambda} \equiv 0$ in a connected component $Z$ of $\Sigma_{\lambda}$ then

$$
\bar{Z} \cap \partial \Omega \cap \partial G^{\lambda}=\emptyset .
$$

Proof. - At any point $z \in \partial \Omega \cap \partial{G_{i}}^{\lambda}$ we have

$$
w_{\lambda}(z)=a_{i}-0>0 .
$$

COROLLARY 2.1. - If $w_{\lambda} \equiv 0$ in a connected component $Z$ of $\Sigma_{\lambda}$, then either $\operatorname{dist}(\bar{Z}, \partial \Omega)>0$ or $Z$ contains a neighbourhood of $\partial \Omega \cap \partial A(Z)$ in $\Omega$, where $A(Z)$ denotes the connected component of $\Omega \cap D_{\lambda}$ containing $Z$.

The next three lemmas ensure the symmetry of $\Omega$, provided the moving plane reaches the critical position $\lambda_{\Omega}$.

LEMMA 2.4. - Either $\beta>0$ or $\beta=0$ and $\frac{\partial^{2} u}{\partial n^{2}}>0$ on $\partial \Omega$. Hence the statement of Step 4 in Section 2.2 is true for $\Omega$ and we can find $\varepsilon_{0}>0$ such that $u>0$ in $\left\{x \in \Omega \mid \operatorname{dist}(x, \partial \Omega)<2 \varepsilon_{0}\right\}$.

Proof. - Since $u \geqslant 0$ in $\Omega$, we immediately see that either $\beta>0$, or $\beta=0$ and $\frac{\partial^{2} u}{\partial n^{2}} \geqslant 0$ on $\partial \Omega$. If Lemma 2.4 were false, following the proof of Step 4 of Section 2.2 we obtain $|\nabla u| \equiv 0$ and $\left|D^{2} u\right|=\frac{\partial^{2} u}{\partial n^{2}} \equiv 0$ on $\partial \Omega$. This implies that the function

$$
\bar{u}(x)= \begin{cases}u(x) & \text { for } x \in \Omega \backslash G, \\ 0 & \text { for } x \in \mathbb{R}^{N} \backslash \bar{\Omega}\end{cases}
$$

belongs to $C^{2}\left(\mathbb{R}^{N} \backslash G\right)$ and solves (4) in $\mathbb{R}^{N} \backslash G$. On the other hand, the result we already proved in Section 2.2 says $\bar{u}$ has to be strictly positive.

LEMMA 2.5. - If $\lambda \in\left[\lambda_{\Omega}, d_{\Omega}\right)$ and $w_{\lambda} \equiv 0$ in a connected component $Z$ of $\Sigma_{\lambda}$ which contains a neighbourhood of $\partial \Omega \cap \partial A(Z)$ in $\Omega$, then $\lambda=\lambda_{\Omega}$ and $\Omega$ is symmetric with respect to $T_{\lambda}$.

Proof. - Note that for $\lambda \in\left[\lambda_{\Omega}, d_{\Omega}\right)$ the set $S:=\partial \Omega \cap \partial A(Z)$ is connected and its reflexion with respect to $T_{\lambda}$ is contained in $\bar{\Omega}$. We shall prove that $S^{\lambda} \subset \partial \Omega$, which is 
the desired symmetry property. Set

$$
H=\left\{z \in S \mid z^{\lambda} \in \partial \Omega\right\} .
$$

We are going to show that $H=S$. First, $H$ is clearly not empty, since it contains the set $T_{\lambda} \cap S$. Second, the set $H$ is open in $S$. To prove this, fix $z^{0} \in H$ and $\varepsilon \in\left(0, \varepsilon_{0}\right)$, such that $B_{\varepsilon}\left(z^{0}\right) \cap A(Z) \subset Z$; here $\varepsilon_{0}$ is the number from the previous lemma. By Lemma 2.4, if $z \in\left(B_{\varepsilon}\left(z^{0}\right) \cap S\right) \backslash H$, then $w_{\lambda}(z)>0$, which implies $z \notin \bar{Z}$ - a contradiction (see Fig. 6). We infer that $B_{\varepsilon}(z) \cap S \subset H$, that is, $H$ is open. Finally, it is clear that $S \backslash H$ is open in $S$, and we conclude that $H=S$.

LEMMA 2.6. - If $\lambda_{0} \leqslant \lambda_{\Omega}$, then $w_{\lambda_{\Omega}} \equiv 0$ in some connected component $Z$ of $\Sigma_{\lambda_{\Omega}}$, which contains a neighbourhood of $\partial \Omega \cap \partial A(Z)$ in $\Omega$.

Proof. - To construct $Z$ such that $w_{\lambda_{\Omega}} \equiv 0$ in $Z$ we repeat the proof of Step 8, Section 2.2. Then it is obvious from the construction that $\partial Z \cap \partial \Omega \neq \emptyset$, and Corollary 2.1 yields Lemma 2.6.

Proof of Theorem 2 (bounded domains). - We shall only sketch this proof, as all its ingredients have already appeared above. We adapt the proof in Section 2.2 to the case of a bounded domain $\Omega$. Step 1 is again the initialising step, in which we now prove that $w_{\lambda} \geqslant 0$ in $\Sigma_{\lambda}$ for $\lambda$ smaller, but sufficiently close to $d_{\Omega}$ (this is easy, by using the fact that the maximum principle holds for any linear strictly elliptic operator with bounded coefficients, provided the measure of the domain is sufficiently small). Step 2 is the same, since Corollary 2.1 and Lemma 2.5 prevent $w_{\lambda} \equiv 0$ in a connected component of $\Sigma_{\lambda}$ and, in particular, in a right neighbourhood of $T_{\lambda}$, for $\lambda>\max \left\{d, \lambda_{\Omega}, \lambda_{\star}\right\}$. By using this fact, we can prove (as in Step 3) that $\lambda_{0} \leqslant \max \left\{d, \lambda_{\Omega}\right\}$, replacing the convergence to infinity of $\left\{x^{(m)}\right\}$ by convergence to $\partial \Omega$. It is important to note that the points $x^{(m)}$ can neither lie on $\partial \Omega$ (since $w_{\lambda} \geqslant 0$ there), nor tend to a point on $\partial \Omega \cap \partial G_{i}^{\lambda}$ (since $w_{\lambda}=a_{i}>0$ at such points). The only difference with Step 3 is that here $\left\{x^{(m)}\right\}$ may tend to a point on $\partial \Omega \cap T_{\lambda}$. In this case we obtain a contradiction by using Lemma 2.4 and the argument at the end of Step 5.

Theorem 6 is already proved, since we can always suppose that we have $\lambda_{\Omega} \geqslant x_{1}^{0}=d$ (if necessary, take a moving plane coming from the left). This means that the moving plane reaches the critical position $\lambda_{\Omega}$, so Lemmas 2.5 and 2.6 permit us to obtain Theorem 6.

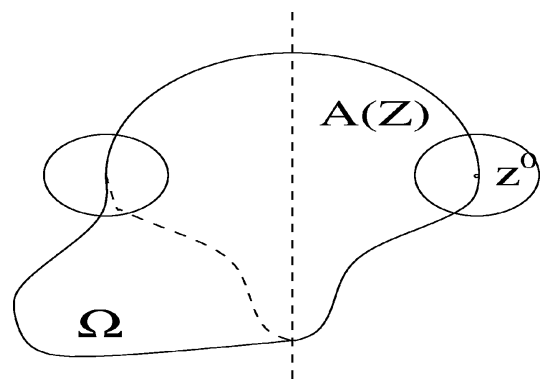

Fig. 6. The contradiction in Lemma 2.5. 
There are no other modifications in Steps 4-7, from which we infer that $\lambda_{0} \leqslant$ $\max \left\{\lambda_{\Omega}, \lambda_{\star}\right\}$. If $\lambda_{\Omega} \geqslant \lambda_{\star}$, then it follows from Lemmas 2.5 and 2.6 that $\Omega$ is symmetric with respect to $T_{\lambda_{\Omega}}$. By applying the argument in Steps 9-10 to the set $Z$ given by Lemma 2.6, we see that $G$ is also symmetric (note that the symmetry of $\Omega$ implies $G^{\lambda_{\Omega}} \subset \Omega$ ). If $\lambda_{\star} \geqslant \lambda_{\Omega}$, as in Steps 8-9 we obtain $w_{\lambda_{\star}} \equiv 0$ in a connected component $Z$ of $\Sigma_{\lambda_{\star}}$, such that $\partial Z \backslash T_{\lambda_{\star}} \subset \partial G$. Since $\Omega \backslash G$ is connected, we can repeat the topological argument in Step 10 to conclude that $\Omega \backslash G$ is symmetric.

Proof of Theorem 5. - If $G$ is a ball, as in Section 2.2 we obtain $u<a$ in $\Omega \backslash G$. If $\Omega$ is a ball and $\lambda_{\star}>\lambda_{\Omega}$, we obtain a contradiction as in the last proof. If $\lambda_{\star}<\lambda_{\Omega}$, we consider a moving plane coming from the left.

\section{Acknowledgement}

The author is very grateful to Professor J. Serrin for many valuable comments on this paper and for bringing to his attention the two references [9] and [14].

\section{REFERENCES}

[1] Alessandrini G., A symmetry theorem for condensers, Math. Meth. Appl. Sc. 15 (1992) 315-320.

[2] Amick C.J., Fraenkel L.E., Uniqueness of Hill's spherical vortex, Arch. Rat. Mech. Anal. 92 (1986) 91-119.

[3] Aftalion A., Busca J., Radial symmetry for overdetermined elliptic problems in exterior domains, Arch. Rat. Mech. Anal. 143 (1998) 195-206.

[4] Berestycki H., Nirenberg L., On the method of moving planes and the sliding method, Bull. Soc. Brazil Mat. Nova Ser. 22 (1991) 1-37.

[5] Castro A., Shivaji R., Non-negative solutions to a semilinear Dirichlet problem in a ball are positive and radially symmetric, Comm. Partial Differential Equations 14 (8\&9) (1989) 1091-1100.

[6] Gidas B., Ni W.-M., Nirenberg L., Symmetry and related properties via the maximum principle, Comm. Math. Phys. 6 (1981) 883-901.

[7] Gilbarg D., Trudinger N., Elliptic Partial Differential Equations of Second Order, 2nd edn., Springer-Verlag, 1983.

[8] Li C., Monotonicity and symmetry of solutions of fully nonlinear elliptic equations on unbounded domains, Comm. Partial Differential Equations 16 (1991) 585-615.

[9] Pucci P., Serrin J., Zou H., A strong maximum principle and a compact support principle for singular elliptic inequalities, J. Math. Pures Appl. 78 (4) (1999) 769-789.

[10] Reichel W., Radial symmetry by moving planes for semilinear elliptic BVP's on annuli and other non-convex domains, in: Bandle C. et al. (Eds.), Progress in PDE's: Elliptic and Parabolic Problems, Pitman Res. Notes, Vol. 325, pp. 164-182.

[11] Reichel W., Radial symmetry for elliptic boundary-value problems on exterior domains, Arch. Rat. Mech. Anal. 137 (1997) 381-394.

[12] Reichel W., Radial symmetry for an electrostatic, a capillarity and some fully nonlinear overdetermined problems on exterior domains, Z. Anal. Anwendungen 15 (1996) 619635.

[13] Serrin J., A symmetry theorem in potential theory, Arch. Rat. Mech. Anal. 43 (1971) 304 318. 
[14] Serrin J., Zou H., Symmetry of ground states of quasilinear elliptic equations, Arch. Rat. Mech. Anal. 148 (4) (1999) 265-290.

[15] Willms N.B., Gladwell G., Siegel D., Symmetry theorems for some overdetermined boundary-value problems on ring domains, Z. Angew. Math. Phys. 45 (1994) 556-579.

[16] Vazquez J.-L., A strong maximum principle for some quasilinear elliptic equations, Appl. Math. and Optimisation 12 (1984) 191-202. 